\title{
Linking RNA Dysfunction and Neurodegeneration in Amyotrophic Lateral Sclerosis
}

\author{
Sami J. Barmada
}

Published online: 18 February 2015

(C) The American Society for Experimental NeuroTherapeutics, Inc. 2015

\begin{abstract}
The degeneration of motor neurons in amyotrophic lateral sclerosis (ALS) inevitably causes paralysis and death within a matter of years. Mounting genetic and functional evidence suggest that abnormalities in RNA processing and metabolism underlie motor neuron loss in sporadic and familial ALS. Abnormal localization and aggregation of essential RNA-binding proteins are fundamental pathological features of sporadic ALS, and mutations in genes encoding RNA processing enzymes cause familial disease. Also, expansion mutations occurring in the noncoding region of $\mathrm{C} 9 \mathrm{orf72}$ - the most common cause of inherited ALS - result in nuclear RNA foci, underscoring the link between abnormal RNA metabolism and neurodegeneration in ALS. This review summarizes the current understanding of RNA dysfunction in ALS, and builds upon this knowledge base to identify converging mechanisms of neurodegeneration in ALS. Potential targets for therapy development are highlighted, with particular emphasis on early and conserved pathways that lead to motor neuron loss in ALS.
\end{abstract}

Key Words ALS · RNA $\cdot$ RNA binding protein · TDP43 . FUS · C9orf72

\section{Introduction}

Amyotrophic lateral sclerosis (ALS) was initially described over 100 years ago by Jean-Martin Charcot [1], and is now recognized as the most common disease affecting both upper

\section{S. J. Barmada $(\bowtie)$}

Department of Neurology, University of Michigan, 109 Zina Pitcher

Place, 5015 Biomedical Sciences Research Building, SSPC 2200,

Ann Arbor, MI 48109, USA

e-mail:sbarmada@umich.edu and lower motor neurons. Charcot was a self-described empiricist, however, and did not speculate on the cause of the disorder. For decades afterwards, no clear etiology could be identified. In fact, many believed that ALS was not a single disease, but rather a clinical diagnosis that might be caused by any number of separate pathologies, all of which result in the death and degeneration of motor neurons. The realization that up to $20 \%$ of patients with familial ALS (fALS) harbor mutations in the gene encoding superoxide dismutase 1 (SOD1) revolutionized our concept of the disease by demonstrating that ALS is, in fact, a disorder with defined genetic causes [2]. This discovery also led directly to the establishment of cellular and animal models that have proved invaluable for studies of motor neuron vulnerability, disease pathogenesis, and potential therapeutic avenues. Still, clinical trials of therapies devised and tested in SOD1 models have proven disappointing in humans [3]. The poor translation of therapies from animal models to humans with ALS might be owing to improperly powered preclinical studies [4] or basic differences between rodent and human physiology. Alternatively, fALS due to SOD1 mutations may be distinct from the majority of ALS, such that therapies developed for the former are ineffective for the latter.

Mounting genetic evidence suggests that motor neuron degeneration in ALS represents a final common pathway initiated by $\geq 1$ conserved upstream mechanisms, including protein misfolding/aggregation [5-12], RNA misprocessing [10, 13-19], and disruptions in axonal transport [12, 20-24]. These mechanisms are not mutually exclusive, and instead may enhance one another, thereby accentuating neurodegeneration in ALS. The identification of neuronal cytoplasmic inclusions rich in the RNA-binding protein transactive response element DNA/RNA binding protein of $43 \mathrm{kDa}$ (TDP43) provided an important link between two of these mechanisms - protein aggregation and RNA dysfunction [6, 7]. TDP43-positive inclusions are a hallmark of sporadic ALS (sALS) and the 
majority of fALS $[6,7,25,26]$, and mutations in the genes encoding TDP43 (TARDBP) and related RNA binding proteins $[15,16,19,27,28]$ cause fALS, emphasizing the critical importance of RNA processing to the pathogenesis of both sALS and fALS. TDP43 is a member of the heterogenous nuclear ribonuclear protein (hnRNP) family of RNA binding proteins that have diverse and integral functions in RNA metabolism and homeostasis [29]. Mutations in the genes encoding several hnRNPs - hnRNP A1, hnRNP A2/B1 [10], and Matrin 3 [19] — cause fALS or motor neuron disease as part of a multisystem disorder. TDP43 also shares significant structural and functional homology with fused in sarcoma (FUS), an RNA-binding protein that is part of the FET family of RNA/DNA-binding proteins that includes FUS, Ewing sarcoma breakpoint region 1 (EWSR1), and TATA box binding protein-associated factor 15 (TAF15). Supporting the connection between abnormal RNA processing and motor neuron disease, nonsense or missense mutations in FUS, EWSR1, and TAF15 are associated with fALS [30,31]. Moreover, trinucleotide (CAG) expansion mutations in ATXN2, encoding the RNA-binding protein ataxin-2, cause spinocerebellar ataxia or ALS, depending on the number of CAG repeats [32-35], and, in some populations, hexanucleotide $\left(\mathrm{G}_{4} \mathrm{C}_{2}\right)$ expansion mutations in the noncoding region of the $C 9$ orf 72 locus account for up to $40 \%$ of fALS $[17,18]$. Individuals carrying C9orf72 expansion mutations also exhibit RNA-rich nuclear foci, indicative of fundamental abnormalities in RNA metabolism.

Together, these findings make a strong argument for aberrant RNA processing as a primary driver of neurodegeneration in ALS. This review describes the specific aspects of RNA misprocessing implicated in ALS pathogenesis, including differences in RNA expression, splicing, and regulation (Fig. 1), and how these events might lead to motor neuron loss in the disorder. Last, I speculate on converging mechanisms of neurotoxicity that are stimulated by disruptions in RNA processing, and identify pathways that might be targeted to delay or prevent neurodegeneration in ALS.

\section{RNA Expression}

TDP43 binds to and regulates thousands of RNA targets [13, $14,36-38]$, and is thus in a unique position to dramatically affect gene expression on a global scale. The RNA-binding domains of TDP43 and FUS are essential for toxicity in ALS model systems, testifying to the importance of RNA dysregulation in ALS pathogenesis [34, 39-41]. Immunoprecipitation studies in murine brain [14], dissociated primary cortical neurons [37], and human brain [13] and cell lines [13, 38] showed that TDP43 recognizes nearly one-thrid of all transcribed genes by binding to redundant GU-rich sequences [42]. While nuclear TDP43 binds preferentially to distal intronic sequences, cytoplasmic TDP43 recognizes more 3' untranslated region (UTR) binding sites [13]. TDP43 regulates its own pre-mRNA, as well as several others involved in neurodegenerative diseases, including those encoding FUS and progranulin [14]. Interestingly, progranulin haploinsufficiency - caused by nonsense or missense mutations in $G R N$ - causes frontotemporal lobar degeneration with TDP43-positive inclusions (FTLD-TDP) [43], a condition that exhibits characteristic pathological and clinical overlap with ALS [6]. However, GRN mutations rarely cause ALS [43-46], indicating that mutations in different genes may result in convergent molecular pathologies but distinct clinical phenotypes. TDP43 also binds several transcripts involved in RNA splicing, nucleocytoplasmic protein trafficking, synapse formation and function, neurotransmitter metabolism, and neurite development $[13,14,37]$. TDP43 dysfunction, therefore, can disrupt fundamental and ubiquitous processes, as well as neuron-specific pathways.

In healthy neurons, TDP43 is predominantly a nuclear protein, but in ALS TDP43 is excluded from the nucleus and accumulates in cytoplasmic inclusions [26]. Within the nucleus, TDP43 has essential roles in RNA transcription, splicing, and stability $[13,14,47-50]$, and transports select mRNA to localized sites of translation within the cytoplasm [20,51, 52]. In addition to its function in RNA trafficking, cytoplasmic TDP43 also helps regulate RNA translation and homeostasis by sequestering transcripts in RNA granules [53-56]. Thus, the loss of TDP43 function stemming from inadequate nuclear protein, a gain of function arising from the accumulation of cytoplasmic TDP43, or both, might result in RNA misprocessing and subsequent neurodegeneration. Genetic ablation of TDP43 is lethal, causing death of the embryo only a few days after fertilization [57, 58]. In mouse striatum, targeted knockdown of TDP43 by means of a local inoculation with antisense oligonucleotides (ASOs) increased expression of 362 genes and downregulated 239 others [14]. Likewise, reduction of TDP43 expression by RNA interference in cultured mouse primary neurons significantly affected the expression of 204 genes [59]; the lower number in this case may be due to the use of an exon array instead of high-throughput RNA sequencing. Notably, many of the differentially expressed genes identified in these studies are required for neuronal function and maintenance, including neurexin 1 and 3, neuroligin, $N$-methyl-D-aspartate receptor subunit 2A, and voltage-dependent calcium channel 1 [14].

Analogous results were obtained in flies lacking $T B P H$, the Drosophila equivalent of TDP43 [60]. Although TBPH-null flies and those overexpressing wild-type (WT) TDP43 exhibited similar locomotor deficits, high-throughput RNA sequencing revealed a distinct set of dysregulated genes in each case. $T B P H$ knockdown primarily increased gene expression (681/910 differentially expressed transcripts), whereas TDP43-overexpressing flies overwhelmingly demonstrated 


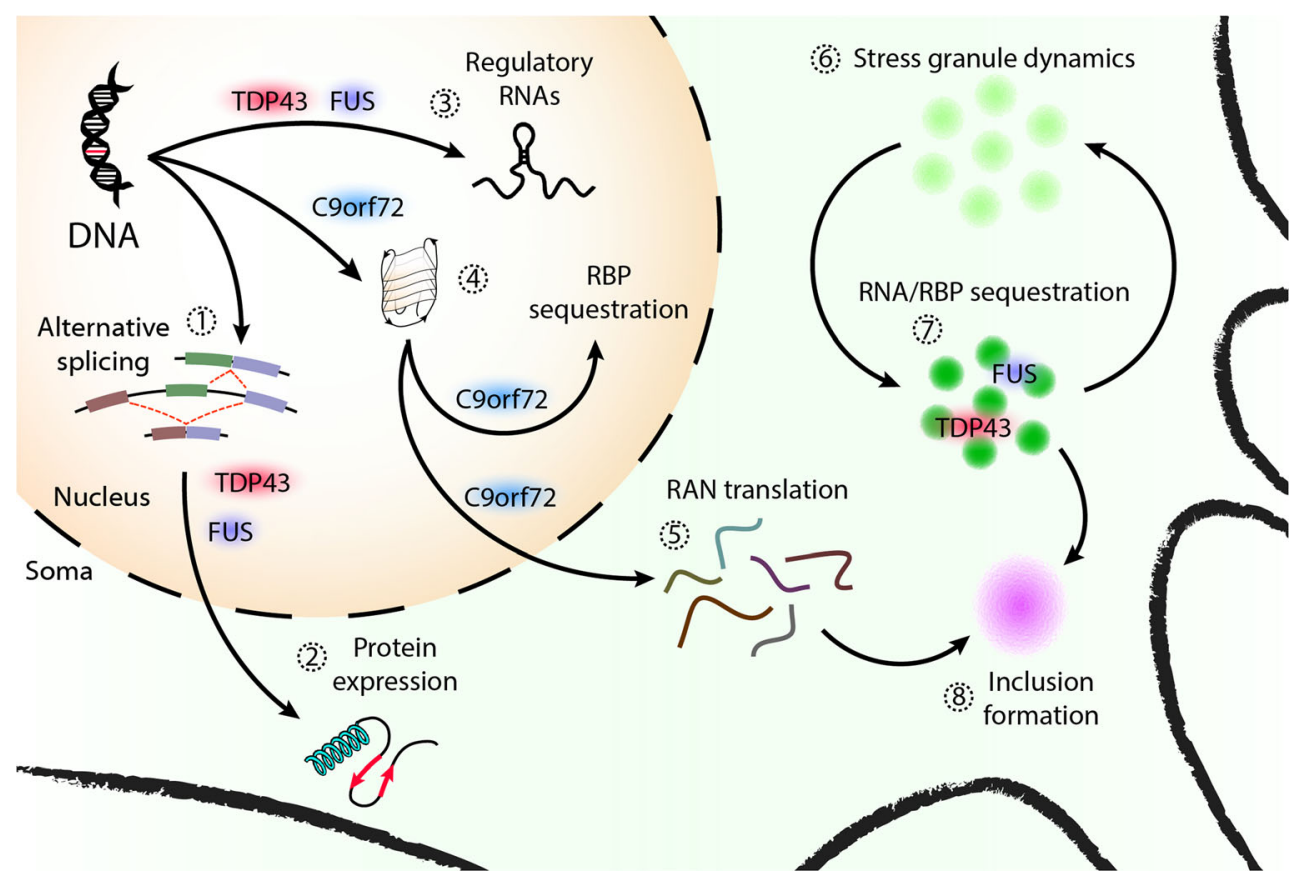

Fig. 1 RNA dysfunction in amyotrophic lateral sclerosis (ALS). Abnormal accumulation or localization of the RNA binding proteins transactive response element DNA/RNA binding protein of $43 \mathrm{kDa}$ (TDP43) or fused in sarcoma (FUS) can affect (1) global splicing machinery and (2) gene expression. (3) In addition, these proteins associate with microRNA (miRNA) assembly machinery, and changes in their levels or distribution are likely to affect the processing of miRNAs and other noncoding regulatory RNAs. (4) Hexanucleotide $\mathrm{G}_{4} \mathrm{C}_{2}$ repeats in C9orf72 form G-quadruplex secondary structures that sequester essential

gene downregulation (464/623 transcripts). Only 79 genes out of a total of 1533 were affected in a conserved manner, arguing against a common pathway accounting for toxicity due to TDP43 loss or gain of function. Consistent with this hypothesis, Arnold et al. [47] demonstrated that many TDP43 targets are dysregulated only upon overexpression of TDP43, while others are affected specifically by TDP43 depletion. Similarly, changes in gene expression induced by fALS-associated mutant TDP43 mimic those of TDP43 depletion in some cases, but others are more consistent with overexpression of WT TDP43. The location and density of intronic TDP43 binding sites affect gene expression and splicing [13], such that gene structure and sequence may predict differential expression upon cytoplasmic accumulation or nuclear depletion of TDP43, or, alternatively, TARDBP mutations. These observations suggest that a limited subset of genes harboring a specific pattern of TDP43 binding sites are preferentially affected in ALS, and may be primarily responsible for motor neuron loss in the disorder. Such disease-specific genetic "signatures" of TDP43 binding sites remain unknown, however.

Consistent with the notion that abnormal RNA metabolism underlies neurodegeneration in ALS, disease-causing mutations in related RNA-binding proteins also disrupt RNA processing. Most ALS-associated mutations in FUS involve the nuclear RNA-binding proteins (RBPs). (5) C9orf $72 \mathrm{G}_{4} \mathrm{C}_{2}$ repeats are also translated through repeat-associated non-AUG (RAN) translation, and the resulting dipeptides may have direct neurotoxic effects. (6) TDP43 and FUS participate in the formation of RNA-rich stress granules, and can disrupt physiologic stress granule assembly or disassembly. (7) Abnormal stress granule kinetics may result in the abnormal sequestration of bound RNAs and associated RBPs, and (8) facilitate the irreversible formation of insoluble protein aggregates that are a characteristic feature of ALS

nuclear localization signal $[15,16]$, resulting in deficient nuclear import and cytoplasmic accumulation of the protein [61]. Reductions in FUS induce widespread changes in gene expression and splicing that demonstrate partial overlap with those observed in TDP43-deficient murine primary neurons and human induced pluripotent stem cell (iPSC)-derived neurons [36, 59]. Although TDP43 and FUS display distinct RNA binding patterns, they both strongly affect the expression of genes with long ( $>150 \mathrm{Kbp})$ introns. A minor portion of the genes most prominently downregulated by TDP43 depletion were affected in a similar fashion by FUS knockdown, including neuroligins and neurexins that are essential for neuronal health. Still, genes displaying overlapping patterns of dysregulation represented only a small fraction of the total, with the majority of differentially expressed genes being distinct in each case [36]. In addition, targeted reduction in TDP43 or FUS produced disparate effects in NSC34 mouse motor neuron-like cells [62]. In an animal model of ALS, the overexpression of FUS bearing an ALS-associated mutation (R521C) significantly affected the expression of $>700$ genes [63]. Many of the upregulated genes in this context involved extracellular matrix or immune processes, while downregulated transcripts included those responsible for neuron projections and ion channel functions. These results must be 
interpreted with caution, however, as RNA was isolated from end-stage animals displaying significant neurodegeneration. Moreover, many of the measured effects of FUS depletion or overexpression may not be direct, as FUS binds RNA polymerase II and regulates nucleosome assembly [64, 65], and may therefore indirectly affect global gene expression.

Pathogenic hexanucleotide expansion mutations in C9orf72 are associated with distinct changes in gene expression that only partially overlap with those induced by TDP43 or FUS dysregulation [66-69]. Human iPSCs carrying disease-associated $C 9$ orf 72 expansions exhibited prominent downregulation of several essential genes [68], including those involved in synaptic transmission, cell adhesion, and membrane excitability. Importantly, this effect was strikingly muted in mutant C9orf72 fibroblasts [67], suggesting that many of the differentially expressed genes are unique to neurons. The $\mathrm{C} 9$ orf72 protein is ubiquitously expressed but highly enriched in neurons $[66,68,70]$, with a proposed function in endosomal trafficking [71]. However, targeted knockdown C9orf72 failed to recapitulate the same pattern of gene expression noted in fibroblasts from ALS patient cells carrying C9orf72 mutations, implying that a simple loss of C9orf72 function is unlikely to explain the observed pattern of RNA dysregulation in C9orf72 mutant cells [67]. In addition, C9orf72 knockdown was not associated with adverse effects or neuronal loss in mice receiving ASOs [67]. Knockdown of the mutant $C 9$ orf 72 allele also restored expression for several affected genes in $C 9$ orf 72 -mutant iPSC-derived neurons [66, $68]$, indicating that this therapeutic strategy may prove effective and safe in humans with disease.

Many of the same changes in gene expression noted in murine primary neurons, transgenic animal ALS models, and iPSC-derived neurons have been confirmed in human central nervous system tissue. TDP43 exhibits a conserved RNA binding pattern in human neuroblastoma cell lines (SH-SY5Y), human embryonic stem cells, and postmortem human cortex [13]. Likewise, the RNA targets of FUS are $70 \%$ conserved in mouse and human brain [36]. Despite the distinct cellular heterogeneity typical of both motor cortex and iPSC cultures, several differentially expressed genes were commonly dysregulated in human iPSC-derived motor neurons and motor cortex from ALS patients with C9orf72 expansion mutations [68]. Rabin et al. [72] purified RNA from motor neurons that had been laser-dissected from the spinal cord of patients with sALS and demonstrated a significant enrichment of cellular adhesion genes in alternatively spliced transcripts. A similar pattern was observed in a mouse model of the motor neuron disease spinal muscular atrophy (SMA) [73], suggesting either that these differences might be a secondary phenomenon observed in association with neurodegeneration or that disrupted cell adhesion represents a conserved neurotoxic pathway in ALS and SMA. In support of the latter hypothesis, Sareen et al. [66] also noted an enrichment of differentially expressed cellular adhesion genes in iPSC-derived neurons from patients with $C 9$ orf72 expansions.

Deficiencies in adenosine deaminase, RNA-specific, isoform 2 (ADAR2) have previously been implicated in ALS pathogenesis and the selective vulnerability of motor neurons in this condition [74]. Affected motor neurons in sALS display reduced expression of ADAR2, resulting in abnormal editing of RNA encoding the $\alpha$-amino-3-hydroxy-5-methyl4-isoxazolepropionic acid (AMPA) receptor (GluA2) and intrinsic susceptibility to excitotoxicity [75]. Several key findings link aberrant ADAR2 functioning with the development of motor neuron loss in ALS. First, downregulation of ADAR isoforms has been observed in human iPSC-derived neurons from patients with C9orf72-linked ALS [68], as well as in TDP43-deficient cell lines [38]. Second, abnormal ADARmediated RNA editing was noted in worms lacking TDP-1, the Caenorhabditis elegans ortholog of TDP43 [76]. Third, ADAR2 recognizes the expanded C9orf $72 \mathrm{G}_{4} \mathrm{C}_{2}$ hexanucleotide repeat and is incorporated into RNA-rich nuclear foci characteristic of $C 9$ orf72-linked fALS [68]. Fourth, TDP43 binds directly to ADARl RNA and protein [37, 77]. The ADAR 1 and 2 isoforms share considerable overlap in substrate specificity [78], such that either or both may mediate GluA2 RNA editing. These observations strongly suggest that abnormal RNA editing is a fundamental event in the pathogenesis of sALS and C9orf72-linked fALS, and that ADAR dysfunction likely contributes to selective motor neuron vulnerability in both conditions.

\section{RNA Splicing}

TDP43 and FUS regulate the splicing of hundreds of genes, and abnormalities in either protein have striking and widespread effects on exon usage (Fig. 1). ASO-mediated knockdown of TDP43 altered 779 splicing events in rodent striatum [14], while targeted reduction of FUS through similar means affected 374 splicing events [36]. Depending on where TDP43 and FUS bind in relation to the exon-intron boundaries, exon inclusion may be either enhanced or reduced by deficiencies in TDP43 or FUS $[13,14,36]$, pointing toward complex patterns of splicing regulation, and partially explaining the pleiotropic effects of depletion, accumulation, or mislocalization of each protein.

In fALS due to TARDBP or FUS mutations, both gain-offunction and loss-of-function splicing deficits may underlie neurodegeneration. The majority of splicing changes observed in transgenic mice overexpressing mutant TDP43 were similar to those induced by overexpression of WT TDP43; however, a small proportion showed changes in splicing consistent with loss of TDP43 function [47]. Among the differentially regulated splicing events observed in mutant TDP43 transgenic mice were those involving neurexins 1 and 3, and GluA2transcripts that are either direct targets of TDP43 or are 
processed by gene products that are regulated by TDP43 (i.e., ADAR) $[14,36]$. In contrast to results from mutant TDP43 transgenic mice, overexpression of mutant FUS in an animal model of ALS resulted in the retention of $>1000$ introns, significantly more than with WT FUS overexpression (177 introns) [63]. In addition, mutant FUS dramatically altered the splicing and expression of an essential neuroprotective agent, brainderived neurotrophic factor (BDNF). Exogenous replacement of BDNF rescued dendritic and synaptic abnormalities in mutant FUS-overexpressing primary neurons [63], suggesting that abnormalities in RNA processing can be overcome and neuronal function improved in some instances by manipulating downstream signaling pathways.

\section{MicroRNA}

Independent of their effects on gene expression and splicing, changes in the abundance or localization of TDP43 or FUS might stimulate genome-wide dysfunction by interfering with the biogenesis and processing of noncoding regulatory RNAs such as microRNA (miRNA) [79, 80] (Fig. 1). Consistent with this hypothesis, both TDP43 and FUS were co-purified with the Drosha miRNA processing complex from human cells [81]. Subsequent studies confirmed these interactions [82], and demonstrated that TDP43, but not FUS, associates with Dicer, an essential cytoplasmic miRNA processing complex [83]. TDP43 binds directly to some miRNAs, and silencing of TDP43 significantly reduced the abundance of several miRNAs (miR-132-5p/3p, miR-143-5p/3p, and miR-574-3p) in human cell lines [84], suggesting that TDP43 is involved in the production or maturation of distinct miRNA species. Importantly, patients with ALS exhibited abnormal levels of each of these miRNAs [84], but the direction of change (up- or downregulation) in ALS tissue was inconsistent and varied depending on the source of the tissue (either cerebrospinal fluid or serum). These results have 2 important implications: 1) as with differences in gene expression, the observed changes in miRNA abundance in ALS are consistent with both gain and loss of TDP43 function; and 2) differences in miRNA levels are tissue type-specific, potentially contributing to the selective vulnerability of motor neurons in ALS. Furthermore, miRNA processing is absolutely required for neuronal health, and deficiencies in miRNA biogenesis lead to neurodegeneration $[85,86]$, implying that miRNA dysregulation induced by TDP43 or FUS aggregation or mislocalization may contribute to motor neuron loss in ALS.

\section{RNA Granules}

Both TDP43 and FUS display glutamine- and asparagine-rich domains that enable the proteins to self-aggregate in a regulated fashion [87]. Similar domains are characteristic of yeast prions that reversibly change their conformation, selfaggregate, and propagate this conformation onto natively folded proteins [88]. Prion-like domains are also found in mammalian proteins whose function requires reversible selfaggregation, including proteins that are involved in the formation of stress granules [89], RNA-dense cytoplasmic particles that sequester nonessential mRNAs and prevent their translation when cells are under duress [90]. TDP43 and FUS are incorporated into cytoplasmic stress granules upon exposure to oxidative, heat, or endoplasmic reticulum stress, but return to their normal (predominantly nuclear) localization when the stress is removed [41, 53, 55, 56, 91, 92]. The reversible aggregation of TDP43 and FUS, and the resulting sequestration of any bound RNAs, may be essential to their physiological functions [87]; however, disrupting the precarious balance between aggregate formation and dissolution may also contribute to the irreversible development of inclusions and the pathogenic deposition of RNA binding proteins in ALS [93] (Fig. 1).

Healthy cells subjected to intermittent stress are capable of a normal and reversible response involving the formation and dissociation of stress granules [94]. Prolonged or repeated insults over time may erode the ability of the cell to dissociate stress granules efficiently, altering the kinetics of the reaction and increasing stress granule stability [94, 95]. ALS-linked mutations in TARDBP and FUS disrupt stress granule dynamics, favoring larger and less soluble granules [53, 56]. In addition, valosin-containing protein (VCP) deficiency or chemical inhibition of VCP blocks stress granule disassembly in human cells [96]. Mutations in VCP cause a multisystem proteinopathy consisting of motor neuron disease, frontotemporal dementia, inclusion body myopathy, and Paget's disease of bone [97], suggesting that impaired stress granule dynamics is a conserved thread in these disorders $[10$, 96]. The formation of relatively stable, insoluble foci within the cytoplasm might accelerate self-aggregation of TDP43 and FUS via a prion-like mechanism [98], eventually leading to characteristic skein-like structures or large cytoplasmic inclusions typical of ALS pathology [6].

In support of this hypothesis, genes that regulate the formation of stress granules also modulate TDP43-mediated toxicity in yeast, flies, and primary rodent neurons [99, 100]. Deletion of the RNA-binding domains in TDP43 and FUS both prevents their inclusion in stress granules and reduces their toxicity [34, 39-41]. Moreover, components of stress granules have been detected in TDP43-rich cytoplasmic deposits in spinal neurons of patients with ALS [56, 99, 101], but are less common in cortical neurons [102]. These results may be explained by the preferential accumulation of fulllength TDP43 in spinal neurons of patients with ALS, in contrast to the deposition of carboxy-terminal fragments of TDP43 in cortical neurons [103]. Such fragments lack the first 
RNA recognition motif (RRM1), as well as a portion of the prion-like domain, both of which are required for stress granule formation [102].

In addition to their roles in sequestering RNAs within stress granules, TDP43 and FUS shuttle RNA from the nucleus to the dendrites in an activity-dependent process $[20,51,52$, 104]. Disease-causing mutations in TARDBP reduce the density and mobility of these RNA particles and interfere with their transport into dendrites $[20,51]$. These results suggest that dysregulation of TDP43 or FUS through inherited mutations or acquired mislocalization might inhibit mRNA transport and localized translation, both of which are essential for proper neuronal function.

TDP43 and FUS are also an integral components of nuclear Gems, or Gemini of Cajal, the site of assembly for small nucleolar RNA particles (snRNPs) that participate in splicing [105]. Within these structures, FUS and TDP43 directly interact with survival in motor neuron (SMN), a protein required for Gem formation [106, 107]. Insufficiency of SMN, resulting from $S M N$ deletion or alternative splicing, is the primary cause of SMA, an autosomal dominant disease of lower motor neurons characterized clinically by progressive muscle atrophy and paralysis [108]. As with SMN depletion, lack of FUS or TDP43 disrupts Gem formation [107, 109], and Gems are reduced in neurons and fibroblasts from patients with ALS [107, 110], indicating that disruptions in snRNP assembly and splicing represent a common mechanism of motor neuron degeneration in ALS and SMA.

Upon stimulation by proinflammatory mediators, TDP43 dissociates from nuclear Gems and catalyzes the formation of interleukin (IL) splicing activating compartments, nuclear structures essential for the regulation and processing of cytokine RNAs. In the absence of TDP43, these compartments failed to form properly, resulting in inadequate production of select cytokines, including IL-6 and IL-10, and immune dysregulation [111]. Conversely, overexpression of TDP43 induced excessive cytokine production and central nervous system inflammation [112]. These data suggest that accumulation of TDP43 in sALS and fALS may trigger characteristic neuroinflammation and subsequent neurodegeneration [113].

Armakola et al. [114] identified a novel protective strategy by conducting an unbiased screen for genetic modifiers of TDP43-based toxicity in yeast. One of the top hits from this screen was debranching enzyme 1 , a protein required for the metabolism of intron lariats formed during RNA splicing. In $d b r l$ knockout cells, lariats accumulate and bind TDP43, effectively deactivating TDP43 by reducing its association with its RNA substrates. Similar techniques may be effective in "defusing" cytoplasmic accumulations of TDP43 and FUS and preventing neurotoxic downstream consequences by competitive inhibition of their RNA binding domains.

\section{Sequestration}

Not only do TDP43 and FUS have numerous RNA targets, but they also associate with key protein mediators of RNA processing. Cytoplasmic accumulation and deposition of these proteins can therefore trigger large-scale deficits in RNA metabolism indirectly, by sequestering essential components of RNA transcription, splicing, stability, and transport. For instance, Schwartz et al. [115] demonstrated that nuclear FUS forms complex RNA-rich particles that tightly bind to the carboxy-terminal domain of RNA polymerase II, effectively sequestering the enzyme. In addition, cytoplasmic accumulations of FUS associate with and sequester protein arginine methyltransferase 1 , resulting in deficient methylation and acetylation of nucleosome components [65], thereby affecting global gene expression. TDP43 binds to a diverse set of RNA processing proteins, including several hnRNPs, splicing factors, ribosomal proteins, and nucleosome subunits [77, $82,116]$. Many of the factors associated with TDP43 and FUS are components of cytoplasmic RNA stress granules [poly(A)binding protein 1, cytotoxic granule-associated RNA binding protein] or miRNA processing complexes (Drosha, Dicer). In addition, TDP43 binds Matrin 3, VCP, EWSR1, TAF15, and FUS, all of which can cause fALS when mutated $[9,15,16$, 19, 30, 31].

Abnormal nucleotide repeat expansions in coding and noncoding regions of the genome cause several neurodegenerative or neurodevelopmental disorders, including myotonic dystrophy, Huntington's disease, spinocerebellar ataxias, and fragile X syndrome [117]. When these expansions occur in protein coding sequences, the result is a repeating stretch of amino acids (i.e., glutamine) that makes the protein prone to misfolding and aggregation. Repeat expansions occurring in noncoding sequences often cause toxicity through a distinct mechanism involving the sequestration of vital RNA binding proteins. In myotonic dystrophy, noncoding repeats in $D M P K$ (type 1) or CNBP (type 2) are transcribed into RNAs that accumulate in ribonuclear foci and sequester essential RNA processing factors, including muscleblind-like proteins, CUG triplet repeat RNA binding protein 1; hnRNP H; and Staufen 1 [118]. Several of these proteins associate with TDP43 [77], hinting at conserved mechanisms of cellular dysfunction in myotonic dystrophy and motor neuron disease. Similarly, C9orf72 RNA transcripts harboring $\mathrm{G}_{4} \mathrm{C}_{2}$ expansion mutations form unique G-quadruplex secondary structures and DNA-RNA hybrids that sequester RNA-binding proteins $[119,120]$ (Fig. 1). Many of the proteins bound by $\mathrm{G}_{4} \mathrm{C}_{2}$ repeats are required for normal RNA processing, including nucleolin, ADAR, hnRNP A1, hnRNP H, SC35, and serine/ arginine-rich splicing factor 1 and serine/arginine-rich splicing factor 2 [66, 68, 119, 121, 122], suggesting that neuronal toxicity in ALS might arise from a functional depletion of these essential factors. If so, then blocking the repeat domain 
might prevent downstream toxicity. Su et al. [123] conducted a small molecule screen for compounds capable of binding to the $C 9$ orf $72 \mathrm{G}_{4} \mathrm{C}_{2}$ hexanucleotide repeat and competitively inhibiting the association of this domain with RNA-binding proteins. These compounds effectively prevented the formation of RNA foci in fibroblasts and neurons from patients carrying the $C 9$ orf 72 expansion mutation, suggesting that they may also potently inhibit the sequestration of RNA-binding proteins by $\mathrm{G}_{4} \mathrm{C}_{2}$ repeats. Still, the efficacy of these compounds in preventing neurodegeneration due to pathogenic C9orf72 mutations remains unknown.

\section{Alternative RNA-Based Mechanisms}

Rather than one predominant mechanism explaining neurodegeneration, synergistic dysfunction in each of the pathways described above likely contributes to the totality of motor neuron loss in ALS. In addition, alternative mechanisms that do not involve canonical RNA processing pathways but nonetheless trigger RNA dysregulation may also lead to neurodegeneration. Some of these mechanisms are considered below.

The prion-like domains of TDP43 and FUS are essential for their physiologic function in mediating stress granule assembly, and may also be important for the propagation of neurodegeneration in an RNA-dependent manner. Recombinant, aggregated TDP43 [98], and insoluble TDP43 isolated from the brains of patients with ALS or FTLD-TDP are capable of inducing TDP43 aggregation and cytotoxicity through a self-templated process in cultured cell lines [98, 124]. Propagation of aggregated TDP43 and cell death could be inhibited by treating TDP43 extracts with formic acid, a powerful denaturant, but not by boiling or mild protease treatment, analogous to mammalian prions. Moreover, the newly aggregated TDP43 maintained characteristic biochemical properties reminiscent of prion "strains" originally described in mammalian prion diseases [124, 125]. fALS-associated mutations in TARDBP are clustered within the prion-like domain of TDP43 [27], and enhance the self-aggregation and seeding behavior of TDP43 fragments [126, 127]. Likewise, mutations in the prion-like domain of hnRNP A1 and hnRNP A2/B1 that cause multisystem proteinopathy facilitate self-aggregation of peptides bearing the mutations, and accelerate the aggregation of WT hnRNP isoforms through a seeding mechanism [10]. The RNA-binding ability of TDP43, hnRNP A1, and hnRNP A2/B1 may be key to the propagation of neurodegeneration, as RNA is a potent and necessary cofactor for the conversion of the mammalian prion protein $\operatorname{PrP}^{\mathrm{C}}$ into its pathogenic isoform, $\operatorname{PrP}^{\mathrm{Sc}}$ [128]. Confirming the connection between neurodegeneration and prion-like behavior of RNA-binding proteins, algorithms designed to identify proteins harboring prion-like domains revealed an enrichment of RNA binding proteins $[28,31]$, many of which have since been implicated in ALS and FTLD pathogenesis [30, 129].

The $C 9$ orf 72 hexanucleotide $\mathrm{G}_{4} \mathrm{C}_{2}$ repeat is located within the first intron of the locus, in a noncoding region, and yet is translated into polypeptides through a unique pathway known as repeat-associated non-AUG dependent (RAN) translation [130-133] (Fig. 1). The mechanism of RAN translation likely involves inefficient RNA splicing and stalling of the ribosome as it scans the hexanucleotide repeat, leading to inappropriate translation without a start codon $[134,135]$. The $\mathrm{G}_{4} \mathrm{C}_{2}$ repeat is translated in all reading frames in both the sense and antisense strands, resulting in several different dipeptides that accumulate specifically within the cells of patients carrying pathogenic $(>22)$ repeat lengths. These dipeptides are toxic to cells in vitro and in vivo [69, 136-138], causing cell death in some cases by disrupting RNA processing within nucleoli [69], or by altering the function of essential proteins such as Unc119 [137], a trafficking factor required for axon development [139]. Moreover, the sequestration of RNA binding proteins by expanded $\mathrm{G}_{4} \mathrm{C}_{2}$ RNA may be synergistic with the toxicity induced by RAN translation products [138]: many of the proteins bound by $\mathrm{G}_{4} \mathrm{C}_{2}$ RNA catalyze the nuclear export of RNA, potentially facilitating cytoplasmic RAN translation of expanded C9orf72 repeats and accentuating toxicity [121].

Trinucleotide (CAG) expansions in the coding region of ataxin-2 are associated with spinocerebellar ataxia type 2, a disease marked by abnormal balance, coordination, neuropathy, and muscle weakness. The CAG repeats are translated into long stretches of glutamine, and while normal individuals have $<22$ glutamines, patients with spinocerebellar ataxia type 2 harbor $>33$ [32]. Intermediate length ataxin-2 repeats-between 22 and 33 glutamine residues - are associated with an enhanced risk of developing ALS [34, 35]. Ataxin-2 directly interacts with TDP43, and intermediate-length ataxin-2 polyglutamine repeats facilitate this association and promote incorporation of TDP43 into cytoplasmic stress granules. In addition, intermediate-length ataxin- 2 polyglutamine repeats accelerate phosphorylation and cleavage of TDP43, and stimulate caspase-3 activation in human motor neurons [140]. Together, these modifications likely enhance motor neuron degeneration and symptoms of ALS in patients carrying intermediate-length ataxin-2 polyglutamine expansions.

Cytoplasmic mislocalization of TDP43 and FUS are characteristic pathologic features in the majority of patients with ALS, and mounting evidence indicates that accumulation of either protein in the cytoplasm is neurotoxic [61, 141]. Deletion of the RNA-binding domain prevents the toxicity of cytoplasmic TDP43 and FUS [39-41], and $d b r l$ deficiency creates a similar effect by flooding cells with intron lariats that compete for TDP43's RNA binding sites [114]. A primary abnormality in nucleocytoplasmic transport would be expected to stimulate mislocalization of TDP43 and FUS, and, as a 
result, disrupt RNA processing. Consistent with this hypothesis, disease-associated mutations in TARDBP and FUS promote cytoplasmic mislocalization of TDP43 and FUS, and enhance neurotoxicity [61, 141]. TDP43 also regulates the expression of Ran, a master mediator of nucleocytoplasmic transport [37, 142], and Ran expression was reduced to $60 \%$ of normal levels in FTLD-TDP cortex [143]. Furthermore, dominant-negative Ran mutations induce cytoplasmic TDP43 deposition and neurotoxicity, while overexpression of WT Ran in a neuron model of FTLD-TDP prevented TDP43 mislocalization and improved survival [143]. Ran was also identified in a proteomic screen for RNA-interacting proteins that specifically bind expanded $C 9$ orf $72 \mathrm{G}_{4} \mathrm{C}_{2}$ hexanucleotide repeats [68], providing a possible mechanism for TDP43 mislocalization in C9orf72-linked ALS. Thus, Ran could be a central and early instigator of TDP43 mislocalization and subsequent neurodegeneration in ALS, and might be a key target of future therapies.

TDP43 and FUS are both capable of binding to and regulating the expression of their own mRNAs through elegant negative feedback loops, and any disruption of these processes could potentially lead to protein deposition and dysfunctional RNA processing. TDP43 binds to the 3'UTR of its transcript, triggering alternative splicing and RNA degradation through exosome- or nonsense-mediated RNA decay pathways [14, 49]. Binding of FUS to its pre-mRNA enhances exon 7 exclusion, thereby generating a shift in the reading frame and uncovering a premature stop codon in exon 8 of the spliced transcript, which is then degraded by nonsense-mediated RNA decay [36, 144]. Importantly, these processes require nuclear localization of TDP43 and FUS for alternative splicing to occur. Cytoplasmic TDP43 and FUS deposition or irreversible stress granule formation would interfere with negative feedback, triggering a vicious cycle of increased protein expression and enhanced cytoplasmic accumulation. As total and cytoplasmic TDP43 levels are directly proportional to toxicity $[141,145,146]$, this phenomenon would be expected to result eventually in neuronal dysfunction and neurodegeneration. The autoregulatory capacity of TDP43 and FUS also represents a potential caveat for investigations involving their overexpression, as the subsequent downregulation of endogenous protein might cause toxicity [147], and confound the interpretation of changes in gene expression or splicing induced by overexpression. Still, exogenously expressed TDP43 is functional and can compensate for endogenous TDP43 deficiency [148], arguing against neurodegeneration due to loss of endogenous TDP43 in overexpression models.

Owing to its unique ability to remove misfolded proteins or dysfunctional organelles, macroautophagy (or autophagy) is emerging as a promising potential therapeutic mechanism for neurodegenerative diseases [149]. Insoluble protein inclusions are a shared pathologic hallmark of neurodegenerative diseases, and genetic and pharmacologic means of stimulating autophagy have proven effective in reducing the burden of misfolded proteins and improving outcomes in cellular and animal models of disease [150-152]. Recent evidence also suggests that autophagy is involved in the turnover of cytoplasmic stress granules through a pathway termed granulophagy [96]. Autophagic induction, therefore, may be useful for enhancing the dissociation of stress granules, preventing irreversible deposition of TDP43 and FUS within the cytoplasm, and restoring functional TDP43 and FUS autoregulation. Indeed, small molecule inducers of autophagy have shown promise in a rodent primary neuron model of ALS, and in human iPSC-derived motor neurons and astrocytes from patients with ALS [145]. In animal models of FTLD-TDP, stimulation of autophagy also reduces symptoms of disease and the accumulation of TDP43 [152]. Autophagy is absolutely necessary for neuronal health [153], and mutations in genes encoding autophagy mediators (p62, ubiquilin-2, VCP) cause fALS [5, 8, 9]. Moreover, essential components of the autophagy pathway are regulated at the RNA level by TDP43 [154]. Abnormalities in autophagy, triggered by inherited mutations in autophagy proteins or changes in TDP43 expression or localization, might compromise granulophagy and prevent stress granule dissociation (Fig. 1). Conversely, stimulation of autophagy may improve granulophagy in affected neurons, in the process restoring TDP43 homeostasis and reversing deficits in RNA metabolism.

\section{Conclusions}

Proper regulation of RNA transcription, splicing, transport, degradation, and translation are critical for maintaining the health of metabolically active cells such as neurons. Nuclear clearing and cytoplasmic accumulation of the RNA binding proteins TDP43 and FUS are fundamental features of both sALS and fALS, and these changes cause genome-wide disruptions in nearly all categories of RNA processing. The extensive nature of RNA dysregulation in ALS represents a potential barrier for newly devised therapeutics and may partially explain the poor response of patients with ALS to previously tested therapies. However, these observations also imply that global RNA dysregulation may be a late and converging mechanism in ALS pathogenesis. Treatments that can act early in the sequence of events by preventing or reversing the accumulation or cytoplasmic deposition of TDP43 or FUS, dissolving $\mathrm{G}_{4} \mathrm{C}_{2}$ RNA foci, or blocking RAN translation may allow affected neurons in ALS to restore protein and RNA homeostasis and therefore slow neuronal dysfunction and death. With these goals in mind, the identification of genetic or physiological biomarkers that predict progression to ALS will be crucial for instituting effective therapies before 
RNA processing has been irreversibly subverted and neurodegeneration has begun.

Acknowledgments I thank Stacey Jacobey and Elizabeth Tank for their critical appraisal of the manuscript. This work was supported by National Institutes of Health - National Institute for Neurological Disorders and Stroke, the ALS Therapy Alliance, the Babcox Foundation, the Protein Folding Diseases Initiative at the University of Michigan, and the Department of Neurology at the University of Michigan.

Required Author Forms Disclosure forms provided by the authors are available with the online version of this article.

\section{References}

1. Charcot JM, Joffory A. Deux cas d'atrophie musculaire progressive avec lesions de la substance grise et des faisceaux antero-lateraux de la moelle epiniere. Arch Physiol Neurol Pathol 1869;2:744-754.

2. Rosen DR, Siddique T, Patterson D, et al. Mutations in $\mathrm{Cu} / \mathrm{Zn}$ superoxide dismutase gene are associated with familial amyotrophic lateral sclerosis. Nature 1993;362:59-62.

3. Turner B, Talbot K. Transgenics, toxicity and therapeutics in rodent models of mutant SOD1-mediated familial ALS. Prog Neurobiol 2008;85:94-134.

4. Gill A, Kidd J, Vieira F, Thompson K, Perrin S. No benefit from chronic lithium dosing in a sibling-matched, gender balanced, investigator-blinded trial using a standard mouse model of familial ALS. PLoS ONE 2009;4:e6489.

5. Deng H-X, Chen W, Hong S-T, et al. Mutations in UBQLN2 cause dominant X-linked juvenile and adult-onset ALS and ALS/dementia. Nature 2011;477:211-215.

6. Neumann M, Sampathu DM, Kwong LK, et al. Ubiquitinated TDP43 in frontotemporal lobar degeneration and amyotrophic lateral sclerosis. Science 2006;314:130-133.

7. Arai T, Hasegawa M, Akiyama H, et al. TDP-43 is a component of ubiquitin-positive tau-negative inclusions in frontotemporal lobar degeneration and amyotrophic lateral sclerosis. Biochem Biophys Res Commun 2006;351:602-611.

8. Fecto F, Yan J, Vemula SP, et al. SQSTM1 mutations in familial and sporadic amyotrophic lateral sclerosis. Arch Neurol 2011;68:14401446.

9. Johnson JO, Mandrioli J, Benatar M, et al. Exome sequencing reveals VCP mutations as a cause of familial ALS. Neuron 2010;68: 857-864.

10. Kim HJ, Kim NC, Wang Y-D, et al. Mutations in prion-like domains in hnRNPA2B1 and hnRNPA1 cause multisystem proteinopathy and ALS. Nature 2013;495:467-473.

11. Fang Y-S, Tsai K-J, Chang Y-J, et al. Full-length TDP-43 forms toxic amyloid oligomers that are present in frontotemporal lobar dementia-TDP patients. Nat Commun 2014;5:4824.

12. Bosco DA, Morfini G, Karabacak NM, et al. Wild-type and mutant SOD1 share an aberrant conformation and a common pathogenic pathway in ALS. Nat Neurosci 2010;13:1396-1403.

13. Tollervey JR, Curk T, Rogelj B, et al. Characterizing the RNA targets and position-dependent splicing regulation by TDP-43. Nat Neurosci 2011;14:452-458.

14. Polymenidou M, Lagier-Tourenne C, Hutt KR, et al. Long premRNA depletion and RNA missplicing contribute to neuronal vulnerability from loss of TDP-43. Nat Neurosci 2011;14:459-468.

15. Vance C, Rogelj B, Hortobágyi T, et al. Mutations in FUS, an RNA processing protein, cause familial amyotrophic lateral sclerosis type 6. Science 2009;323:1208-1211.
16. Kwiatkowski TJ, Bosco DA, Leclerc AL, et al. Mutations in the FUS/TLS gene on chromosome 16 cause familial amyotrophic lateral sclerosis. Science 2009;323:1205-1208.

17. Renton AE, Majounie E, Waite A, et al. A hexanucleotide repeat expansion in C9ORF72 is the cause of chromosome 9p21-linked ALS-FTD. Neuron 2011;72:257-268.

18. Dejesus-Hernandez M, Mackenzie IR, Boeve BF, et al. Expanded GGGGCC hexanucleotide repeat in noncoding region of C9ORF72 causes chromosome 9p-linked FTD and ALS. Neuron 2011;72:245256.

19. Johnson JO, Pioro EP, Boehringer A, et al. Mutations in the Matrin 3 gene cause familial amyotrophic lateral sclerosis. Nat Neurosci 2014; 17:664-666

20. Alami NH, Smith RB, Carrasco MA, et al. Axonal transport of TDP-43 mRNA granules is impaired by ALS-causing mutations. Neuron 2014;81:536-543.

21. Julien JP, Côté F, Collard JF. Mice overexpressing the human neurofilament heavy gene as a model of ALS. Neurobiol Aging 1995; 16:487-490.

22. Ligon LA, LaMonte BH, Wallace KE, et al. Mutant superoxide dismutase disrupts cytoplasmic dynein in motor neurons. Neuroreport 2005;16:533-536.

23. Wu C-H, Wu C-H, Fallini C, et al. Mutations in the profilin 1 gene cause familial amyotrophic lateral sclerosis. Nature 2012;488:499 503.

24. Novarino G, Fenstermaker AG, Zaki MS, et al. Exome sequencing links corticospinal motor neuron disease to common neurodegenerative disorders. Science 2014;343:506-511.

25. Ravits J, Appel S, Baloh RH, et al. Deciphering amyotrophic lateral sclerosis: What phenotype, neuropathology and genetics are telling us about pathogenesis. Amyotroph Lateral Scler Frontotemporal Degener 2013;14(S1):5-18.

26. Neumann M. Molecular neuropathology of TDP-43 proteinopathies. Int J Mol Sci 2009;10:232-246.

27. Barmada SJ, Finkbeiner S. Pathogenic TARDBP mutations in amyotrophic lateral sclerosis and frontotemporal dementia: disease-associated pathways. Rev Neurosci 2010;21:251-272.

28. King OD, Gitler AD, Shorter J. The tip of the iceberg: RNA-binding proteins with prion-like domains in neurodegenerative disease. Brain Res 2012;1462:61-80.

29. Smith R, Rathod RJ, Rajkumar S, Kennedy D. Nervous translation, do you get the message? A review of mRNPs, mRNA-protein interactions and translational control within cells of the nervous system. Cell Mol Life Sci 2014;71:3917-3937.

30. Couthouis J, Hart MP, Erion R, et al. Evaluating the role of the FUS/ TLS-related gene EWSR1 in amyotrophic lateral sclerosis. Hum Mol Genet 2012;21:2899-2911.

31. Couthouis J, Hart MP, Shorter J, et al. A yeast functional screen predicts new candidate ALS disease genes. Proc Natl Acad Sci U S A 2011;108:20881-20890.

32. Pulst SM, Nechiporuk A, Nechiporuk T, et al. Moderate expansion of a normally biallelic trinucleotide repeat in spinocerebellar ataxia type 2. Nat Genet 1996;14:269-276.

33. Lattante S, Millecamps S, Stevanin G, et al. Contribution of ATXN2 intermediary polyQ expansions in a spectrum of neurodegenerative disorders. Neurology 2014;83:990-995.

34. Elden AC, Kim H-J, Hart MP, et al. Ataxin-2 intermediate-length polyglutamine expansions are associated with increased risk for ALS. Nature 2010;466:1069-1075.

35. Lee $\mathrm{T}$, Li YR, Ingre $\mathrm{C}$, et al. Ataxin-2 intermediate-length polyglutamine expansions in European ALS patients. Hum Mol Genet 2011;20:1697-1700.

36. Lagier-Tourenne C, Polymenidou M, Hutt KR, et al. Divergent roles of ALS-linked proteins FUS/TLS and TDP-43 intersect in processing long pre-mRNAs. Nat Neurosci 2012;15:1488-1497. 
37. Sephton CF, Cenik C, Kucukural A, et al. Identification of neuronal RNA targets of TDP-43-containing ribonucleoprotein complexes. J Biol Chem 2011;286:1204-1215.

38. Xiao S, Sanelli T, Dib S, et al. RNA targets of TDP-43 identified by UV-CLIP are deregulated in ALS. Mol Cell Neurosci 2011;47:167180.

39. Voigt A, Herholz D, Fiesel FC, et al. TDP-43-mediated neuron loss in vivo requires RNA-binding activity. PLoS ONE 2010;5:e12247.

40. Ihara R, Ihara R, Matsukawa K, et al. RNA binding mediates neurotoxicity in the transgenic Drosophila model of TDP-43 proteinopathy. Hum Mol Genet 2013;22:4474-4484.

41. Daigle JG, Daigle JG, Lanson NA, et al. RNA binding ability of FUS regulates neurodegeneration, cytoplasmic mislocalization and incorporation into stress granules associated with FUS carrying ALS-linked mutations. Hum Mol Genet 2012;22:1193-1205.

42. Buratti E, Baralle FE. Characterization and functional implications of the RNA binding properties of nuclear factor TDP-43, a novel splicing regulator of CFTR exon 9. J Biol Chem 2001;276:3633736343.

43. Cruts M, Gijselinck I, van der Zee J, et al. Null mutations in progranulin cause ubiquitin-positive frontotemporal dementia linked to chromosome 17q21. Nature 2006;442:920-924.

44. Baker M, Mackenzie IR, Pickering-Brown SM, et al. Mutations in progranulin cause tau-negative frontotemporal dementia linked to chromosome 17. Nature 2006;442:916-919.

45. Schymick JC, Yang Y, Andersen PM, et al. Progranulin mutations and amyotrophic lateral sclerosis or amyotrophic lateral sclerosisfrontotemporal dementia phenotypes. J Neurol Neurosurg Psychiatry 2006;78:754-756.

46. Cannon A, Fujioka S, Rutherford NJ, et al. Clinicopathologic variability of the GRN A9D mutation, including amyotrophic lateral sclerosis. Neurology 2013;80:1771-1777.

47. Arnold ES, Ling S-C, Huelga SC, et al. ALS-linked TDP-43 mutations produce aberrant RNA splicing and adult-onset motor neuron disease without aggregation or loss of nuclear TDP-43. Proc Natl Acad Sci U S A 2013;110:E736-E745.

48. Ayala YM, Pantano S, D'Ambrogio A, et al. Human, Drosophila, and C. elegans TDP43: Nucleic acid binding properties and splicing regulatory function. J Mol Biol 2005;348:575-588.

49. Ayala YM, De Conti L, Avendaño-Vázquez SE, et al. TDP-43 regulates its mRNA levels through a negative feedback loop. EMBO J 2010;30:277-288.

50. Avendano-Vazquez SE, Dhir A, Bembich S, Buratti E, Proudfoot N, Baralle FE. Autoregulation of TDP-43 mRNA levels involves interplay between transcription, splicing, and alternative polyA site selection. Genes Dev 2012;26:1679-1684.

51. Liu-Yesucevitz L, Lin AY, Ebata A, et al. ALS-linked mutations enlarge TDP-43-enriched neuronal RNA granules in the dendritic arbor. J Neurosci 2014;34:4167-4174.

52. Wang I-F, Wu L-S, Chang H-Y, Shen C-KJ. TDP-43, the signature protein of FTLD-U, is a neuronal activity-responsive factor. J Neurochem 2008;105:797-806.

53. McDonald KK, Aulas A, Destroismaisons L, et al. TAR DNAbinding protein 43 (TDP-43) regulates stress granule dynamics via differential regulation of G3BP and TIA-1. Hum Mol Genet 2011;20:1400-1410.

54. Dewey CM, Cenik B, Sephton CF, et al. TDP-43 is directed to stress granules by sorbitol, a novel physiological osmotic and oxidative stressor. Mol Cell Biol 2011;31:1098-1108.

55. Colombrita C, Zennaro E, Fallini C, et al. TDP-43 is recruited to stress granules in conditions of oxidative insult. J Neurochem 2009;111:1051-1061.

56. Liu-Yesucevitz L, Bilgutay A, Zhang Y-J, et al. Tar DNA binding protein-43 (TDP-43) associates with stress granules: analysis of cultured cells and pathological brain tissue. PLoS ONE 2010;5: e13250.
57. Sephton CF, Good SK, Atkin S, et al. TDP-43 is a developmentally regulated protein essential for early embryonic development. J Biol Chem 2010;285:6826-6834.

58. Wu L-S, Cheng W-C, Hou S-C, Yan Y-T, Jiang S-T, Shen C-KJ. TDP-43, a neuro-pathosignature factor, is essential for early mouse embryogenesis. Genesis 2010;48:56-62.

59. Honda D, Ishigaki S, Iguchi Y, et al. The ALS/FTLD-related RNAbinding proteins TDP-43 and FUS have common downstream RNA targets in cortical neurons. FEBS Open Bio 2013;4:1-10.

60. Hazelett DJ, Chang J-C, Lakeland DL, Morton DB. Comparison of parallel high-throughput RNA sequencing between knockout of TDP-43 and its overexpression reveals primarily nonreciprocal and nonoverlapping gene expression changes in the central nervous system of Drosophila. G3 (Bethesda) 2012;2:789-802.

61. Dormann D, Rodde R, Edbauer D, et al. ALS-associated fused in sarcoma (FUS) mutations disrupt Transportin-mediated nuclear import. EMBO J 2010;29:2841-2857.

62. Colombrita C, Onesto E, Megiorni F, et al. TDP-43 and FUS RNAbinding proteins bind distinct sets of cytoplasmic messenger RNAs and differently regulate their post-transcriptional fate in motoneuron-like cells. J Biol Chem 2012;287:15635-15647.

63. Qiu H, Lee S, Shang Y, et al. ALS-associated mutation FUS-R521C causes DNA damage and RNA splicing defects. J Clin Invest 2014;124:981-999.

64. Schwartz JC, Ebmeier CC, Podell ER, Heimiller J, Taatjes DJ, Cech TR. FUS binds the CTD of RNA polymerase II and regulates its phosphorylation at Ser2. Genes Dev 2012;26:2690-2695.

65. Tibshirani M, Tradewell ML, Mattina KR, et al. Cytoplasmic sequestration of FUS/TLS associated with ALS alters histone marks through loss of nuclear protein arginine methyltransferase 1 . Hum Mol Genet 2015;24:773-786.

66. Sareen D, O'Rourke JG, Meera P, et al. Targeting RNA foci in iPSCderived motor neurons from ALS patients with a C9ORF72 repeat expansion. Sci Transl Med 2013;5:208ra149-208ra149.

67. Lagier-Tourenne C, Baughn M, Rigo F, et al. Targeted degradation of sense and antisense C9orf72 RNA foci as therapy for ALS and frontotemporal degeneration. Proc Natl Acad Sci U S A 2013;110: E4530-E4539.

68. Donnelly CJ, Zhang P-W, Pham JT, et al. RNA toxicity from the ALS/FTD C9ORF72 expansion is mitigated by antisense intervention. Neuron 2013;80:415-428.

69. Kwon I, Xiang S, Kato M, et al. Poly-dipeptides encoded by the C9orf72 repeats bind nucleoli, impede RNA biogenesis, and kill cells. Science 2014;345:1139-1145.

70. Suzuki N, Maroof AM, Merkle FT, et al. The mouse C9ORF72 ortholog is enriched in neurons known to degenerate in ALS and FTD. Nat Neurosci 2013;16:1725-1727.

71. Farg MA, Sundaramoorthy V, Sultana JM, et al. C9ORF72, implicated in amytrophic lateral sclerosis and frontotemporal dementia, regulates endosomal trafficking. Hum Mol Genet 2014;23:35793595.

72. Rabin SJ, Kim JMH, Baughn M, et al. Sporadic ALS has compartment-specific aberrant exon splicing and altered cellmatrix adhesion biology. Hum Mol Genet 2009;19:313-328.

73. Zhang Z, Lotti F, Dittmar K, et al. SMN deficiency causes tissuespecific perturbations in the repertoire of snRNAs and widespread defects in splicing. Cell 2008;133:585-600.

74. Hideyama T, Teramoto S, Hachiga K, Yamashita T, Kwak S. Cooccurrence of TDP-43 mislocalization with reduced activity of an RNA editing enzyme, ADAR2, in aged mouse motor neurons. PLoS ONE 2012;7:e43469.

75. Yamashita T, Kwak S. The molecular link between inefficient GluA2 Q/R site-RNA editing and TDP-43 pathology in motor neurons of sporadic amyotrophic lateral sclerosis patients. Brain Res 2014;1584:28-38. 
76. Saldi TK, Ash PE, Wilson G, et al. TDP-1, the Caenorhabditis elegans ortholog of TDP-43, limits the accumulation of doublestranded RNA. EMBO J 2014;33:2947-2966.

77. Freibaum BD, Chitta RK, High AA, Taylor JP. Global analysis of TDP-43 interacting proteins reveals strong association with RNA splicing and translation machinery. J Proteome Res 2010;9:11041120 .

78. Wong SK, Sato S, Lazinski DW. Substrate recognition by ADAR1 and ADAR2. RNA 2001;7:846-858.

79. Gomes A, Nolasco S, Soares H. Non-coding RNAs: Multi-tasking molecules in the cell. Int J Mol Sci 2013;14:16010-16039.

80. Gascon E, Gao F-B. The emerging roles of microRNAs in the pathogenesis of frontotemporal dementia-amyotrophic lateral sclerosis (FTD-ALS) spectrum disorders. J Neurogenet 2014;28:30-40.

81. Gregory RI, Yan K-P, Amuthan G, et al. The microprocessor complex mediates the genesis of microRNAs. Nature 2004;432:235240.

82. Ling S-C, Albuquerque CP, Han JS, et al. ALS-associated mutations in TDP-43 increase its stability and promote TDP-43 complexes with FUS/TLS. Proc Natl Acad Sci U S A 2010;107:13318-13323.

83. Kawahara Y, Mieda-Sato A. TDP-43 promotes microRNA biogenesis as a component of the Drosha and Dicer complexes. Proc Natl Acad Sci U S A 2012;109:3347-3352.

84. Freischmidt A, Müller K, Ludolph AC, Weishaupt JH. Systemic dysregulation of TDP-43 binding microRNAs in amyotrophic lateral sclerosis. Acta Neuropathol Commun 2013;1:42.

85. Haramati S, Chapnik E, Sztainberg Y, et al. miRNA malfunction causes spinal motor neuron disease. Proc Natl Acad Sci U S A 2010;107:13111-13116.

86. Schaefer A, O'Carroll D, Tan CL, et al. Cerebellar neurodegeneration in the absence of microRNAs. J Exp Med 2007;204:15531558 .

87. Udan $\mathrm{M}$, Baloh $\mathrm{RH}$. Implications of the prion-related $\mathrm{Q} / \mathrm{N}$ domains in TDP-43 and FUS. Prion 2011;5:1-5.

88. Chernova TA, Wilkinson KD, Chernoff YO. Physiological and environmental control of yeast prions. FEMS Microbiol Rev 2014;38: 326-344.

89. Gitler AD, Shorter J. RNA-binding proteins with prion-like domains in ALS and FTLD-U. Prion 2011;5:179-187.

90. Anderson P, Kedersha N. Stress granules: The Tao of RNA triage. Trends Biochem Sci 2008;33:141-150.

91. Yasuda K, Zhang H, Loiselle D, Haystead T, Macara IG, Mili S. The RNA-binding protein Fus directs translation of localized mRNAs in APC-RNP granules. J Cell Biol 2013;203:737-746.

92. Baron DM, Kaushansky LJ, Ward CL, et al. Amyotrophic lateral sclerosis-linked FUS/TLS alters stress granule assembly and dynamics. Mol Neurodegener 2013;8:30.

93. Li YR, King OD, Shorter J, Gitler AD. Stress granules as crucibles of ALS pathogenesis. J Cell Biol 2013;201:361-372.

94. Higashi S, Kabuta T, Nagai Y, Tsuchiya Y, Akiyama H, Wada K. TDP-43 associates with stalled ribosomes and contributes to cell survival during cellular stress. J Neurochem 2013;126:288-300.

95. Parker SJ, Meyerowitz J, James JL, et al. Endogenous TDP-43 localized to stress granules can subsequently form protein aggregates. Neurochem Int 2012;60:415-424.

96. Buchan JR, Kolaitis R-M, Taylor JP, Parker R. Eukaryotic stress granules are cleared by autophagy and Cdc48/VCP function. Cell 2013;153:1461-1474.

97. Watts GDJ, Wymer J, Kovach MJ, et al. Inclusion body myopathy associated with Paget disease of bone and frontotemporal dementia is caused by mutant valosin-containing protein. Nat Genet 2004;36: 377-381.

98. Furukawa Y, Kaneko K, Watanabe S, Yamanaka K, Nukina N. A seeding reaction recapitulates intracellular formation of Sarkosylinsoluble transactivation response element (TAR) DNA-binding protein-43 inclusions. J Biol Chem 2011;286:18664-18672.
99. Kim H-J, Raphael AR, LaDow ES, et al. Therapeutic modulation of eIF $2 \alpha$ phosphorylation rescues TDP-43 toxicity in amyotrophic lateral sclerosis disease models. Nat Genet 2014;46:152-160.

100. Figley MD, Bieri G, Kolaitis RM, Taylor JP, Gitler AD. Profilin 1 associates with stress granules and ALS-linked mutations alter stress granule dynamics. J Neurosci 2014;34(24): 8083-8097.

101. McGurk L, Lee VM, Trojanowksi JQ, Van Deerlin VM, Lee EB, Bonini NM. Poly-A binding protein-1 localization to a subset of TDP-43 inclusions in amyotrophic lateral sclerosis occurs more frequently in patients harboring an expansion in C9orf72. J Neuropathol Exp Neurol 2014;73:837-845.

102. Bentmann E, Neumann M, Tahirovic S, Rodde R, Dormann D, Haass C. Requirements for stress granule recruitment of fused in sarcoma (FUS) and TAR DNA-binding protein of $43 \mathrm{kDa}$ (TDP43). J Biol Chem 2012;287:23079-23094.

103. Igaz LM, Kwong LK, Xu Y, et al. Enrichment of C-terminal fragments in TAR DNA-binding protein-43 cytoplasmic inclusions in brain but not in spinal cord of frontotemporal lobar degeneration and amyotrophic lateral sclerosis. Am J Pathol 2008;173:182-194.

104. Fujii R, Takumi T. TLS facilitates transport of mRNA encoding an actin-stabilizing protein to dendritic spines. J Cell Sci 2005;118: 5755-5765.

105. Carvalho T, Almeida F, Calapez A, Lafarga M, Berciano MT, Carmo-Fonseca $\mathrm{M}$. The spinal muscular atrophy disease gene product, SMN: A link between snRNP biogenesis and the Cajal (coiled) body. J Cell Biol 1999;147:715-728.

106. Tsuiji H, Iguchi Y, Furuya A, et al. Spliceosome integrity is defective in the motor neuron diseases ALS and SMA. EMBO Mol Med 2013;5:221-234

107. Yamazaki T, Chen S, Yu Y, et al. FUS-SMN protein interactions link the motor neuron diseases ALS and SMA. Cell Rep 2012;2:799806.

108. Lefebvre S, Bürglen L, Reboullet S, et al. Identification and characterization of a spinal muscular atrophy-determining gene. Cell 1995;80:155-165.

109. Shan X, Chiang P-M, Price DL, Wong PC. Altered distributions of Gemini of coiled bodies and mitochondria in motor neurons of TDP-43 transgenic mice. Proc Natl Acad Sci U S A 2010;107: 16325-16330.

110. Ishihara T, Ariizumi Y, Shiga A, et al. Decreased number of Gemini of coiled bodies and U12 snRNA level in amyotrophic lateral sclerosis. Hum Mol Genet 2013;22:4136-4147.

111. Lee S, Lee TA, Lee E, et al. Identification of a subnuclear body involved in sequence-specific cytokine RNA processing. Nat Commun 2015;6:5791.

112. Herman AM, Khandelwal PJ, Rebeck GW, Moussa CE-H. Wild type TDP-43 induces neuro-inflammation and alters APP metabolism in lentiviral gene transfer models. Exp Neurol 2012;235:297305.

113. Evans MC, Couch Y, Sibson N, Turner MR. Inflammation and neurovascular changes in amyotrophic lateral sclerosis. Mol Cell Neurosci 2013;53:34-41.

114. Armakola M, Higgins MJ, Figley MD, et al. Inhibition of RNA lariat debranching enzyme suppresses TDP-43 toxicity in ALS disease models. Nat Genet 2012;44:1-10.

115. Schwartz JC, Wang X, Podell ER, Cech TR. RNA seeds higherorder assembly of FUS protein. Cell Rep 2013;5:918-925.

116. Chi Y-H, Semmes OJ, Jeang K-T. A proteomic study of TAR-RNA binding protein (TRBP)-associated factors. Cell Biosci 2011;1:9.

117. Li L-B, Bonini NM. Roles of trinucleotide-repeat RNA in neurological disease and degeneration. Trends Neurosci 2010;33:292298.

118. Meola G, Cardani R. Myotonic dystrophies: An update on clinical aspects, genetic, pathology, and molecular pathomechanisms. Biochim Biophys Acta 2014 May 29 [Epub ahead of print]. 
119. Haeusler AR, Donnelly CJ, Periz G, et al. C9orf72 nucleotide repeat structures initiate molecular cascades of disease. Nature 2015;507: 195-200.

120. Reddy K, Schmidt MHM, Geist JM, et al. Processing of double-Rloops in (CAG).(CTG) and C9orf72 (GGGGCC).(GGCCCC) repeats causes instability. Nucleic Acids Res 2014;42:10473-10487.

121. Cooper-Knock J, Walsh MJ, Higginbottom A, et al. Sequestration of multiple RNA recognition motif-containing proteins by $\mathrm{C} 9$ orf 72 repeat expansions. Brain 2014;137(Pt 7):2040-2051.

122. Lee Y-B, Chen H-J, Peres JN, et al. Hexanucleotide repeats in ALS/ FTD form length-dependent RNA foci, sequester RNA binding proteins, and are neurotoxic. Cell Rep 2013;5:1178-1186.

123. Su Z, Zhang Y, Gendron TF, et al. Discovery of a biomarker and lead small molecules to target $\mathrm{r}(\mathrm{GGGGCC})$-associated defects in c9FTD/ALS. Neuron 2014;83:1043-1050.

124. Nonaka T, Masuda-Suzukake M, Arai T, et al. Prion-like properties of pathological TDP-43 aggregates from diseased brains. Cell Rep 2013;4:124-134.

125. Aguzzi A, Rajendran L. The transcellular spread of cytosolic amyloids, prions, and prionoids. Neuron 2009;64:783-790.

126. Guo W, Chen Y, Zhou X, et al. An ALS-associated mutation affecting TDP-43 enhances protein aggregation, fibril formation and neurotoxicity. Nat Struct Mol Biol 2011;18:822-830.

127. Zhu L, Xu M, Yang M, et al. An ALS-mutant TDP-43 neurotoxic peptide adopts an anti-parallel $\beta$-structure and induces TDP-43 redistribution. Hum Mol Genet 2014;23:6863-6877.

128. Deleault NR, Lucassen RW, Supattapone S. RNA molecules stimulate prion protein conversion. Nature 2003;425:717-720.

129. Mackenzie IRA, Neumann M. FET proteins in frontotemporal dementia and amyotrophic lateral sclerosis. Brain Res 2012;1462:4043.

130. Mori K, Weng S-M, Arzberger T, et al. The C9orf72 GGGGCC repeat is translated into aggregating dipeptide-repeat proteins in FTLD/ALS. Science 2013;339:1335-1338.

131. Ash PEA, Bieniek KF, Gendron TF, et al. Unconventional translation of C9ORF72 GGGGCC expansion generates insoluble polypeptides specific to c9FTD/ALS. Neuron 2013;77:639-646.

132. Zu T, Gibbens B, Doty NS, et al. Non-ATG-initiated translation directed by microsatellite expansions. Proc Natl Acad Sci U S A 2011;108:260-265.

133. Zu T, Liu Y, Bañez-Coronel M, et al. RAN proteins and RNA foci from antisense transcripts in C9ORF72 ALS and frontotemporal dementia. Proc Natl Acad Sci U S A 2013;110:E4968-E4977.

134. Todd PK, Oh SY, Krans A, et al. CGG repeat-associated translation mediates neurodegeneration in fragile $\mathrm{X}$ tremor ataxia syndrome. Neuron 2013;78:440-455.

135. Kochetov AV, Palyanov A, Titov II, Grigorovich D, Sarai A, Kolchanov NA. AUG_hairpin: prediction of a downstream secondary structure influencing the recognition of a translation start site. BMC Bioinformatics 2007;8:318.

136. Mizielinska S, Grönke S, Niccoli T, et al. C9orf72 repeat expansions cause neurodegeneration in Drosophila through arginine-rich proteins. Science 2014;345:1192-1194.

137. May S, Hornburg D, Schludi MH, et al. C9orf72 FTLD/ALSassociated Gly-Ala dipeptide repeat proteins cause neuronal toxicity and Unc119 sequestration. Acta Neuropathol 2014;128:485-503.
138. Wen X, Tan W, Westergard T, et al. Antisense proline-arginine RAN dipeptides linked to C9ORF72-ALS/FTD form toxic nuclear aggregates that initiate in vitro and in vivo neuronal death. Neuron 2014;84:1213-1225.

139. Knobel KM, Davis WS, Jorgensen EM, Bastiani MJ. UNC-119 suppresses axon branching in C. elegans. Development 2001;128: 4079-4092.

140. Hart MP, Gitler AD. ALS-associated ataxin 2 polyQ expansions enhance stress-induced caspase 3 activation and increase TDP-43 pathological modifications. J Neurosci 2012;32:9133-9142.

141. Barmada SJ, Skibinski G, Korb E, Rao EJ, Wu JY, Finkbeiner S. Cytoplasmic mislocalization of TDP-43 is toxic to neurons and enhanced by a mutation associated with familial amyotrophic lateral sclerosis. J Neurosci 2010;30:639-649.

142. Hutten S, Kehlenbach RH. CRM1-mediated nuclear export: to the pore and beyond. Trends Cell Biol 2007;17:193-201.

143. Ward ME, Taubes A, Chen R, et al. Early retinal neurodegeneration and impaired Ran-mediated nuclear import of TDP-43 in progranulin-deficient FTLD. J Exp Med 2014;177:311.

144. Zhou Y, Liu S, Liu G, Oztürk A, Hicks GG. ALS-associated FUS mutations result in compromised FUS alternative splicing and autoregulation. PLoS Genet 2013;9:e1003895.

145. Barmada SJ, Serio A, Arjun A, et al. Autophagy induction enhances TDP43 turnover and survival in neuronal ALS models. Nat Chem Biol 2014; 10:677-685.

146. Janssens J, Wils H, Kleinberger G, et al. Overexpression of ALSassociated p.M337V human TDP-43 in mice worsens disease features compared to wild-type human TDP-43 mice. Mol Neurobiol 2013;48:22-35.

147. Igaz LM, Kwong LK, Lee EB, et al. Dysregulation of the ALSassociated gene TDP-43 leads to neuronal death and degeneration in mice. J Clin Invest 2011;121:726-738.

148. Xu Y-F, Prudencio M, Hubbard JM, et al. The pathological phenotypes of human TDP-43 transgenic mouse models are independent of downregulation of mouse Tdp-43. PLoS ONE 2013;8:e69864.

149. Banerjee R, Beal MF, Thomas B. Autophagy in neurodegenerative disorders: pathogenic roles and therapeutic implications. Trends Neurosci 2010;33:541-549.

150. Tsvetkov AS, Miller J, Arrasate M, Wong JS, Pleiss MA, Finkbeiner S. A small-molecule scaffold induces autophagy in primary neurons and protects against toxicity in a Huntington disease model. Proc Natl Acad Sci U S A 2010;107:16982-16987.

151. Hetz C, Thielen P, Matus S, et al. XBP-1 deficiency in the nervous system protects against amyotrophic lateral sclerosis by increasing autophagy. Genes Dev 2009;23:2294-2306.

152. Wang I-F, Guo B-S, Liu Y-C, et al. Autophagy activators rescue and alleviate pathogenesis of a mouse model with proteinopathies of the TAR DNA-binding protein 43. Proc Natl Acad Sci U S A 2012;109: 15024-15029.

153. Hara T, Nakamura K, Matsui M, et al. Suppression of basal autophagy in neural cells causes neurodegenerative disease in mice. Nat Cell Biol 2006;441:885-889.

154. Bose JK, Huang C-C, Shen C-KJ. Regulation of autophagy by neuropathological protein TDP-43. J Biol Chem 2011;286:4444144448 . 